\title{
Punção estromal anterior na descompensação corneana secundária à iridosquise - Relato de caso e revisão da literatura
}

\author{
Anterior stromal puncture in bullouskeratopathy secondary to iridoschisis - \\ Case reportand literature review
}

\author{
Bruno Machado Fontes ${ }^{1}$ \\ Lênio Souza Alvareng'a ${ }^{2}$ \\ Luís Antonio Vieira ${ }^{2}$ \\ Luciene Barbosa de Souza ${ }^{3}$ \\ Denise de Freitas ${ }^{4}$
}

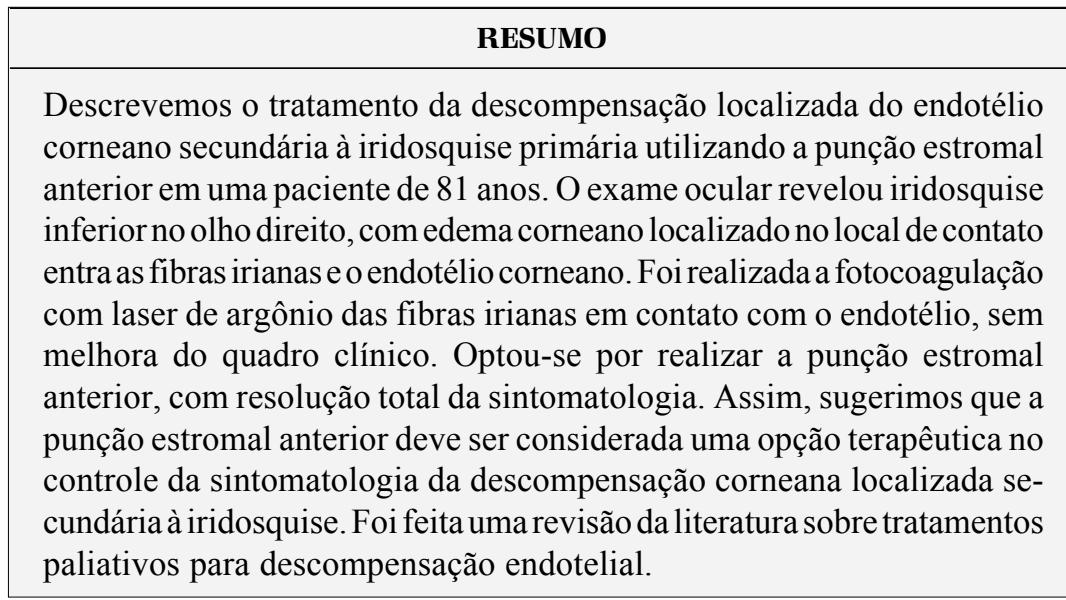

Descritores: Córnea; Endotélio da córnea; Doenças da íris; Assistência paliativa; Edema; Relato de caso
Trabalho realizado no Setor de Doenças Externas Oculares e Córnea; Departamento de Oftalmologia da Universidade Federal de São Paulo - UNIFESP.

Médico - Residente do Departamento de Oftalmologia da Universidade Federal de São Paulo - UNIFESP.

${ }^{2}$ Doutor em Oftalmologia e médico colaborador do Setor de Doenças Externas Oculares e Córnea, Departamento de Oftalmologia da Universidade Federal de São Paulo - UNIFESP.

${ }^{3}$ Doutora em Oftalmologia, professora do curso de PósGraduação em Oftalmologia e chefe do Setor de Doenças Externas Oculares e Córnea, Departamento de Oftalmologia da Universidade Federal de São Paulo UNIFESP.

${ }^{4}$ Livre-Docente em Oftalmologia e professora do curso de Pós-Graduação em Oftalmologia, Departamento de Oftalmologia da Universidade Federal de São Paulo UNIFESP.

Endereço para correspondência: Bruno Machado Fontes Rua Botucatu, 822 São Paulo (SP) CEP 04023-000

E-mail: brunofontes@oftalmo.epm.br

Recebido para publicação em 23.09.2002

Aceito para publicação em 06.03.2003

\section{INTRODUÇÃ̃O}

A iridosquise é uma anormalidade incomum na qual há dissolução localizada do estroma iriano ${ }^{(1)} \mathrm{com}$ posterior separação em duas camadas e desestruturação da camada anterior, causando a formação de fibras ${ }^{(2)}$ que ficam soltas na câmara anterior. Geralmente é bilateral, ocorre mais em idosos e tem uma apresentação setorial com uma inexplicada predileção pela porção inferior da íris ${ }^{(3)}$. Não há formação de buracos irianos e a pupila apresenta-se centrada e reagente aos estímulos ${ }^{(3)}$. Associação com glaucoma ocorre em aproximadamente $50 \%$ dos casos, sendo mais comum o glaucoma de ângulo estreito ${ }^{(1-5)}$. Casos relatados na literatura associam a iridosquise ainda à idade avançada, córnea guttata, ceratocone, disfunção endotelial, traumas, anormalidades congênitas e ceratite intersticial por sífilis ${ }^{(1,-9)}$. A iridosquise pode estar associada aos sintomas decorrentes da descompensação endotelial ${ }^{(1-2,4-5,9)}$.

Dentre outros, são propostos na literatura como tratamentos paliativos para a perda da função endotelial, com conseqüente ceratopatia bolhosa, a punção estromal anterior ${ }^{(10-13)}$, o uso de lentes de contato terapêuticas ${ }^{(14-17)}$, uso tópico de substâncias hiperosmóticas como solução salina a $5 \%^{(11)}$, cauterização da camada de Bowman ${ }^{(11)}$, ceratectomia fototerapêutica (PTK) com o uso do excimer laser(17-20), realização de recobrimento conjuntival ${ }^{(21-22)}$, transplante de membrana 
amniótica $^{(23-24)}$ e transplante lamelar ${ }^{(25-26)}$, sendo o transplante penetrante de córnea considerado o único tratamento definitivo $^{(17-19,22,27)}$.

A punção estromal anterior é usada com sucesso no tratamento de erosões epiteliais recorrentes e na ceratopatia bolhosa ${ }^{(10-12)}$, principalmente quando as lesões são localizadas e não se encontram no eixo visual. O mecanismo de ação exato não é totalmente conhecido, sendo o mais provável a indução de cicatrização, adesão epitelial e fibrose subepitelial criando uma barreira à formação das bolhas, o que impede a ruptura das mesmas e a exposição das terminações nervosas ${ }^{(10-13)}$.

No caso relatado propomos a realização da punção estromal anterior como tratamento alternativo e eficaz no alívio dos sintomas decorrentes da descompensação endotelial localizada secundária à iridosquise.

\section{RELATO DE CASO}

Paciente de 81 anos do sexo feminino, branca, compareceu ao ambulatório de doenças externas oculares e córnea do Departamento de Oftalmologia da UNIFESP com queixa de baixa acuidade visual em ambos os olhos, dor e sensação de corpo estranho no olho direito há 3 meses.

$\mathrm{O}$ exame inicial mostrou acuidade visual com a melhor correção de conta dedos a 2 metros em olho direito e 0,3 em olho esquerdo. As anormalidades reveladas pela biomicroscopia (Figura1) foram guttata na córnea central (escavações circulares, amareladas e reflexivas com pigmentos finos na superfície endotelial com aspecto de "placa batida") e catarata nuclear em ambos os olhos. No olho direito verificou-se também hiperemia conjuntival leve difusa, edema corneano principalmente inferior com dobras no estroma posterior, neovasos superficiais, bolhas epiteliais com ceratite ponteada, presença de iridosquise na região inferior da íris com cordoalhas livres

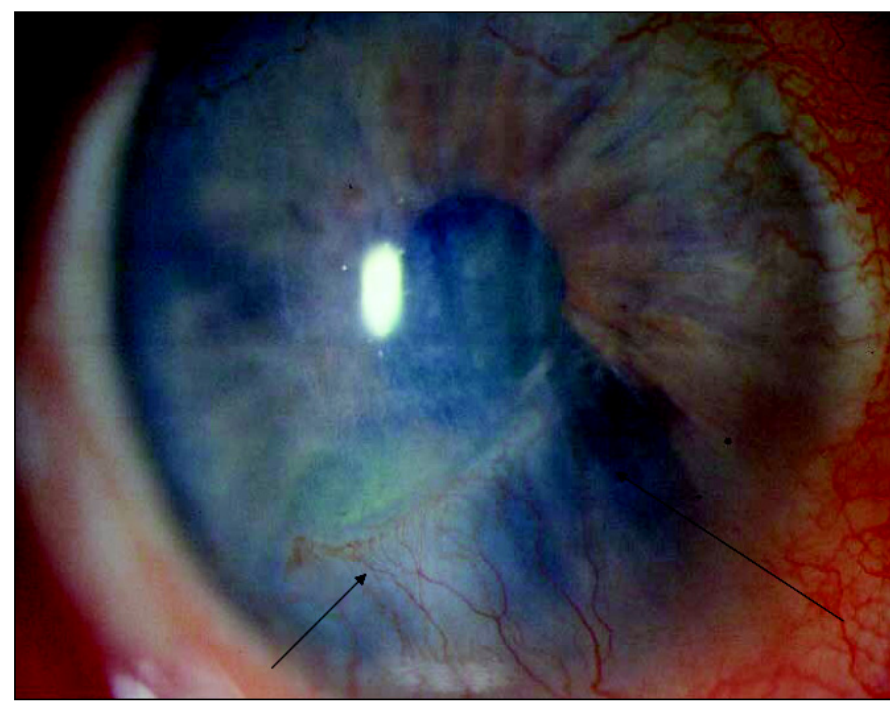

Biomicroscopia inicial mostrando iridosquise inferior (seta longa) e edema corneano (seta curta) tocando o endotélio corneano e deposição de restos de material iriano. A pressão intra-ocular e a fundoscopia mostraramse dentro dos parâmetros da normalidade em ambos os olhos.

A biomicroscopia ultra-sônica (UBM) do olho direito identificou câmara anterior rasa (profundidade central de 1,76 mm), com fibras irianas livres. A córnea mostrou espessura central de 804 micra com descontinuidade endotelial (Figura 2).

Foi realizada fotocoagulação das fibras irianas que estavam em contato com o endotélio corneano com laser de argônio (30 disparos de $250 \mu \mathrm{m}, 0,75 \mathrm{~W}$ e 0,2 seg de duração) objetivando coagular e retrair as fibras irianas que tocavam o endotélio. A paciente retornou após 2 semanas sem melhora da sintomatologia apesar de não apresentar mais, à biomicroscopia, toque entre as fibras irianas e o endotélio.

Optou-se então por realizar a punção estromal anterior na região edemaciada, conforme descrito por Gomes et $\mathrm{al}^{(10)}$ : instilou-se uma gota de proparacaína a $0,5 \%$ e com o auxílio da lâmpada de fenda foram realizadas múltiplas (aproximadamente 100) punções não confluentes no estroma anterior da região acometida, usando uma agulha estéril (calibre 25). Após o procedimento foi colocada lente de contato terapêutica e prescrito colírio antibiótico (Ofloxacino 0,3\%) 3 vezes ao dia até o retorno. Após uma semana a lente de contato foi retirada, e ao exame biomicroscópico não se detectou presença de bolhas epiteliais sendo observada diminuição da neovascularização corneana. A paciente permaneceu assintomática desde então, tendo sido completados 3 meses de seguimento. A opacidade corneana não foi alterada, assim como a acuidade visual inicial.

\section{DISCUSS $\tilde{A} O$}

A patogênese da iridosquise primária não é totalmente esclarecida, sendo comumente considerada uma atrofia relacionada à

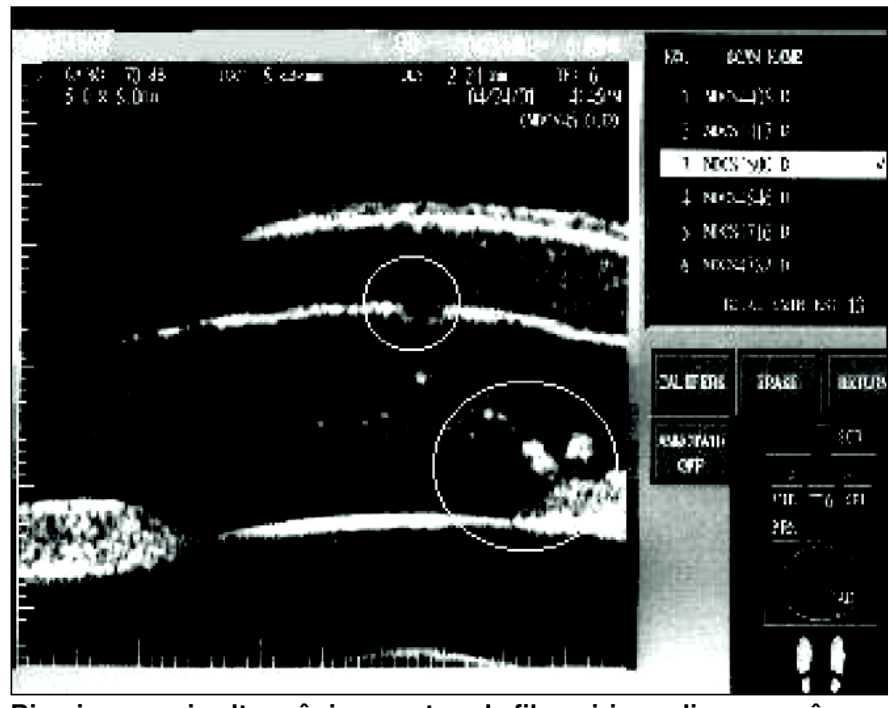

Biomicroscopia ultra-sônica mostrando fibras irianas livres na câmara anterior (círculo maior) e descontinuidade endotelial (círculo menor) 
idade e sem predileção por sexo ${ }^{(1-2,9)}$. Duke-Elder ${ }^{(4)}$ a relacionou à uma "morte natural de tecidos velhos". Estudos realizados não demonstraram anormalidades vasculares, sendo as alterações estromais provavelmente não decorrentes de isquemia ${ }^{(1)}$.

Entre os diagnósticos diferenciais encontramos as síndromes Irido - Corneanas ("ICE Syndromes"), disgenesias mesodérmicas córneo-irianas e iridosquise traumática ${ }^{(3,5,8)}$. Todos estes diagnósticos foram descartados na paciente descrita neste relato, através da história, quadro clínico e exames complementares, sendo a mesma classificada como iridosquise primária.

Alterações corneanas relacionadas à iridosquise primária são incomuns, havendo poucos relatos na literatura de sua associação com ceratopatia bolhosa e cornea guttata ${ }^{(1,5,9)}$. É descrita a correlação topográfica, sendo a parte inferior da córnea na área da iridosquise a primeira a sofrer alteração $0^{(3)}$, provavelmente devido ao contato constante das fibras irianas com o endotélio corneano levando à sua descompensação.

No caso apresentado, as queixas da paciente de sensação de corpo estranho são compatíveis com o quadro clínico apresentado de ceratopatia bolhosa. A presença de córnea guttata bilateral, porém com iridosquise unilateral e edema corneano localizado (correspondendo à região de toque íris/endotélio), indicam que a causa da descompensação provavelmente foi o contato por tempo prolongado entre as fibras irianas e o endotélio corneano.

O tratamento realizado com a fotocoagulação das fibras irianas em contato com o endotélio não resultou em melhora clínica, mesmo tendo sucesso em eliminar o contato entre estas estruturas. O fato de a paciente não apresentar melhora clínica e tampouco biomicroscópica após a fotocoagulação das fibras irianas não sugere, como poderia se supor a princípio, que este procedimento não tenha utilidade. Deve-se ressaltar que considerando a hipótese de descompensação endotelial traumática, a resolução total das alterações só seria possível se tal intervenção ocorresse antes do dano irreversível da função endotelial ter ocorrido. Assim, nesta paciente, a fotocoagulação pode ter sido realizada após lesão importante da função endotelial, mas o procedimento pode ter evitado lesão ainda pior. Pode-se supor ainda, que o tempo entre os dois procedimentos não foi o suficiente para uma eventual expansão das células endoteliais com melhora do edema. No entanto, como a paciente apresentava queixas importantes e quadro clínico de descompensação endotelial, optamos por realizar a punção estromal anterior uma vez que este procedimento parece não interferir com a evolução endotelial ${ }^{(10)}$. A contribuição da fotocoagulação das fibrilas da íris no controle da sintomatologia da descompensação corneana, descrita pela primeira vez na literatura no presente estudo, ainda não está estabelecida e necessitará de trabalhos adicionais.

A punção estromal anterior é um método simples, barato, seguro e efetivo no tratamento sintomático da ceratopatia bolhosa ${ }^{(10-12)}$. O prognóstico em um eventual transplante de córnea futuro não é alterado e os pacientes na maioria das vezes apresentam melhora da sintomatologia dolorosa, podendo no entanto haver decréscimo da acuidade visual ${ }^{(10-11)}$. Este método foi selecionado para o tratamento da paciente relatada pela baixa complexidade e alta eficácia em eliminar os sintomas da ceratopatia bolhosa secundária a outras patologias ${ }^{(10-11)}$. Não há, entretanto, descrição deste método para o tratamento paliativo da descompensação endotelial secundária à iridosquise primária. As outras possibilidades terapêuticas são descritas a seguir, ressaltando-se que para estas também não há descrição de seus usos no tratamento da ceratopatia bolhosa secundária à iridosquise primária.

O uso de lentes de contato terapêuticas fundamenta-se na aplicação de curativos diretamente sobre a superfície ocular ${ }^{(14-16)}$. Estão indicadas como opção no tratamento paliativo da ceratopatia bolhosa, proporcionando alívio dos sintomas e facilitando a cicatrização epitelial. No entanto, seu uso constante está associado com várias complicações entre as quais neovascularização corneana (o que é fator de risco para rejeição de um eventual transplante corneano), piora do edema, ceratites infecciosas e conjuntivite papilar gigante ${ }^{(14-16,28)}$. Necessita de visitas médicas e trocas regulares além de profilaxia antibiótica constante, o que encarece o tratamento ${ }^{(14-16)}$.

A eletrocauterização da camada de Bowman é um método efetivo no controle dos sintomas relacionados à ceratopatia bolhosa, porém pode induzir necrose epitelial, alterar a curvatura corneana (podendo influenciar o astigmatismo pós-transplante) tendo a lesão cicatrização mais lenta que a observada na punção estromal anterior ${ }^{(11)}$.

$\mathrm{O}$ uso da ceratectomia fototerapêutica (PTK) vem sido relatado como tendo sucesso no manejo sintomático de pacientes com prognóstico pobre e sem indicação a um eventual transplante penetrante ${ }^{(17-20)}$. Não se sabe ao certo seu mecanismo, sendo a hipótese de que há melhora da cicatrização com a remoção da membrana basal anormal. Pode haver indução de hipermetropia ${ }^{(11,17-20)}$.

O recobrimento conjuntival é um procedimento cirúrgico indicado no tratamento da ceratopatia bolhosa dolorosa em olhos com baixo potencial visual onde o transplante penetrante de córnea não está indicado e terapias mais simples como uso de lentes de contato terapêuticas ou a punção estromal anterior não obtiveram êxito ${ }^{(21-2)}$. Apresenta como potenciais complicações a perfuração conjuntival, retração do retalho, hemorragia, ptose, cistos mucosos epiteliais e lesão das células tronco do limbo ${ }^{(21-22)}$.

O transplante de membrana amniótica é uma boa opção cirúrgica, obtendo sucesso no alívio doloroso da ceratopatia bolhosa. No entanto, precisa de pessoal treinado na coleta e processamento da mesma o que encarece o tratamento, além da obrigatoriedade de ser feito em ambiente cirúrgico por cirurgião familiarizado com a técnica ${ }^{(23-24)}$.

Há poucos relatos na literatura sobre transplante lamelar no tratamento da descompensação corneana ${ }^{(25-26)}$, sendo descrito como para olhos sem prognóstico visual.

No caso descrito a punção estromal anterior se mostrou uma boa alternativa havendo melhora dos sintomas e manutenção da acuidade visual. O procedimento se mostrou seguro e eficaz. A paciente mantém-se assintomática, tendo completado seguimento de 3 meses. 


\section{AGRADECIMENTOS}

Fabio Dante Garcia e Solange Higashitani.

\section{ABSTRACT}

To describe the treatment of localized bullous keratopathy secondary to iridoschisis with anterior stromal puncture in a 81-year-old female with iridoschisis and localized corneal ede$\mathrm{ma}$, in whom anterior stromal puncture was performed. Ocular examination showed inferior iridoschisis in the right eye, with localized edema in the area of contact between the fibers and the endothelium. Photocoagulation of the fibers was performed but no biomicroscopic changes were observed. Anterior stromal puncture was performed and a complete resolution of symptoms was observed. We suggest that anterior stromal puncture should be considered as an option to reduce the symptoms of patients with localized corneal edema secondary to iridoschisis. Review of the literature about palliative treatments for bullous keratopathy was also performed.

Keywords: Cornea; Endothelium corneal; Iris diseases; Palliative care; Edema; Case report

\section{REFERÊNCIAS}

1. Rodrigues MC, Spaeth GL, Krachmer JH, Laibson PR. Iridoschisis associated with glaucoma and bullous keratopathy. Am J Ophthalmol 1983;95:73-81.

2. Agrawal S, Agrawal J, Agrawal TP. Iridoschisis associated with lens subluxation. J Cataract Refract Surg 2001;27:2044-6.

3. Pearson PA, Amrien JM, Baldwin LB, Smith TJ. Iridoschisis associated with syphilitic interstitial keratitis. Am J Ophthalmol 1989;107:88-90.

4. Duke-Elder S. Disease of the uveal tract. In: Duke-Elder S, editor. System of ophthalmology. St Louis: Mosby; 1966.

5. Weseley AC, Freeman WR. Iridoschisis and the corneal endothelium. Ann Ophthalmol 1983;15:955-9

6. Eiferman RA, Law M, Lane L. Iridoschisis and keratoconus [commented on Cornea 1994;13:545-6]. Cornea 1994;13:78-9.

7. Danias J, Aslanides JM, Eichenbaum JW, Silverman RH, Reinstein DZ, Coleman DJ. Iridoschisis: high frequency ultrasound imaging. Evidence for a genetic defect? Br J Ophthalmol. 1996;80:1063-7.
8. Hersh PS. Iridoschisis following penetrating keratoplasty for keratoconus. Cornea 1994;13:545-6.

9. Streiff EB, Faggioni R, Scouras J. Iridoschisis familial compliqué de cornea guttata. Ophtalmologica 1974;169:411-5.

10. Gomes JA, Haraguchi DK, Zambrano DU, Izquierdo Jr L, Cunha MC, Freitas D. Anterior stromal puncture in the treatment of bullous keratopathy: sixmonth follow-up. Cornea 2001;20:570-2.

11. Cormier G, Brunette I, Boisjoly HM, LeFrançois M, Shi ZH, Guertin MC. Anterior stromal punctures for bullous keratopathy. Arch Ophthalmol 1996;114:654-8.

12. Sridhar MS, Vemuganti GK, Bansal AK, Rao GN. Anterior stromal puncture in bullous keratopathy: a clinicopathologic study. Cornea 2001;20:573-9.

13. Hsu JKW, Rubinfeld RS, Barry P, Jester JV. Anterior stromal puncture immunohistochemical studies in human corneas. Arch Ophthalmol 1993;111: 1057-63.

14. McDermott ML, Chandler JW. Therapeutic uses of contact lenses. Surv Ophthalmol 1989;33:381-94

15. Stein HA, Freeman MI, Stein RM, Maund LD. CLAO - Residents contact lens Curriculum Manual $2^{\text {nd }}$ ed. New York: CLAO; 1999.

16. Bechara SB, Kara-José N. Lentes de contato terapêuticas. In: Belfort Jr R, Kara-José N. Córnea clínica - cirúrgica. São Paulo: Roca; 1997. p. 443-8.

17. Thomann U, Meier-Gibbons F, Schipper I. Phototherapeutic keratectomy for bullous keratopathy. Br J Ophthalmol 1995;79:335-8.

18. Thomann U, Niesen U, Schipper I. Successful phototherapeutic keratectomy for recurrent erosions in bullous keratopathy. J Refract Surg 1996;12:S290-2.

19. Maini R, Sullivan L, Snibson GR, Taylor HR, Loughnan MS. A comparison of different depth ablations in the treatment of painful bullous keratopathy with phototherapeutic keratectomy. Br J Ophthalmol 2001;85:912-5.

20. Lin PY, Wu CC, Lee SM. Combined phototherapeutic keratectomy and therapeutic contact lens for recurrent erosions in bullous keratopathy. Br J Ophthalmol 2001;85:908-11.

21. Alino AM, Perry HD, Kanellopoulos AJ, Donnenfeld ED, Rahn EK. Conjunctival flaps [commented on Ophthalmology 1999;106:210-1]. Ophthalmology 1998;105:1120-3.

22. Wang MX, Karp CL, Selkin RP, Azar DT. Corneal and conjunctival surgery. In: Yanoff M, Duker JS. Ophthalmology. Philadelphia: Mosby International; 1999. p.12-8

23. Mejía LF, Santamaría JP, Acosta C. Symptomatic management of postoperative bullous keratopathy with nonpreserved human amniotic membrane. Cornea 2002;21:342-5.

24. Pires RT, Tseng SC, Prabhasawat P, Puangsricharern V, Maskin SL, Kim JC, et al. Amniotic membrane transplantation for symptomatic bullous keratopathy. Arch Ophthalmol 1999;117:1291-7.

25. Melles GRJ, Lander F, Beekhuis WH, Remeijer L, Binder PS. Posterior lamellar keratoplasty for a case of pseudophakic bullous keratopathy. Am J Ophthalmol 1999; 127:340-1.

26. Koenig SB. Annular keratotomy for the treatment of painful bullous keratopathy. Am J Ophthalmol 1996;121:93-4.

27. Cosar CB, Sridhar MS, Cohen EJ, Held EL, Alvim PTS, Rapuano CJ, et al. Indications for penetrating keratoplasty and associated procedures, 1996-2000. Cornea 2002;21:148-51.

28. Luchs JI, Cohen EJ, Rapuano CJ, Laibson PR. Ulcerative keratitis in bullous keratopathy. Ophthalmology 1997;104:816-22.

\section{Ao enviar um artigo para publicação, leia ATENTAMENTE as instruções para autores, constante no final de cada fascículo.}

